\title{
Adherence to the Mediterranean diet reduces mortality in the Spanish cohort of the European Prospective Investigation into Cancer and Nutrition (EPIC-Spain)
}

\author{
Genevieve Buckland $^{1 *}$, Antonio Agudo ${ }^{1}$, Noemie Travier $^{1}$, José María Huerta ${ }^{2,3}$, Lluís Cirera ${ }^{2,3}$, \\ María-José Tormo ${ }^{2,3,4}$, Carmen Navarro ${ }^{2,3,4}$, María Dolores Chirlaque ${ }^{2,3}$, Conchi Moreno-Iribas ${ }^{3,5,6}$, \\ Eva Ardanaz ${ }^{3,5}$, Aurelio Barricarte ${ }^{3,5}$, Jaione Etxeberria ${ }^{3,5,7}$, Pilar Marin ${ }^{3,5}$, J. Ramón Quirós ${ }^{8}$, \\ María-Luisa Redondo $^{8}$, Nerea Larrañaga ${ }^{3,9}$, Pilar Amiano ${ }^{3,9}$, Miren Dorronsoro ${ }^{3,9}$, Larraitz Arriola ${ }^{3,9}$, \\ Mikel Basterretxea ${ }^{3,9}$, María-José Sanchez ${ }^{3,10}$, Esther Molina ${ }^{3,10}$ and Carlos A. González ${ }^{1}$ \\ ${ }^{1}$ Unit of Nutrition, Environment and Cancer, Cancer Epidemiology Research Programme, Catalan Institute of Oncology \\ (ICO-IDIBELL), Barcelona, Spain \\ ${ }^{2}$ Department of Epidemiology, Murcia Regional Health Council, Murcia, Spain \\ ${ }^{3}$ CIBER Epidemiología y Salud Pública (CIBERESP), Spain \\ ${ }^{4}$ Department of Public Health and Preventive Medicine, University of Murcia, Spain \\ ${ }^{5}$ Public Health Institute of Navarra, Pamplona, Spain \\ ${ }^{6}$ Departments of Pediatrics, Obstetrics and Gynecology, and Preventive Medicine, Universidad Autónoma de Barcelona, \\ Barcelona, Spain \\ ${ }^{7}$ Department of Statistics and O. R.., Universidad Pública de Navarra, Spain \\ ${ }^{8}$ Public Health and Health Planning Directorate, Asturias, Spain \\ ${ }^{9}$ Public Health Department of Gipuzkoa, Basque Government, San Sebastián, Spain \\ ${ }^{10}$ Andalusian School of Public Health, Granada, Spain
}

(Received 14 January 2011 - Revised 14 March 2011 - Accepted 14 March 2011 - First published online 17 May 2011)

\section{Abstract}

Epidemiological studies show that adherence to a Mediterranean diet (MD) increases longevity; however, few studies are restricted to Mediterranean populations or explore the effect of a MD pattern that directly incorporates olive oil. Therefore the relationship between adherence to the MD and mortality was studied within the the Spanish cohort of the European Prospective Investigation into Cancer and Nutrition (EPIC-Spain). The EPIC-Spain analysis included 40622 participants (37.7\% males) aged 29-69 years who were recruited from five Spanish regions in 1992-1996. During a mean follow-up of 13.4 years, 1855 deaths were documented: 913 from cancer, 399 from CVD, 425 from other causes and 118 from unknown causes of death. Risk of all-cause and cause-specific mortality was assessed according to the level of adherence to a relative MD (rMED) score, measured using an 18-unit scale incorporating nine selected dietary components. A high compared with a low rMED score was associated with a significant reduction in mortality from all causes (hazard ratio (HR) 0.79; $95 \%$ CI 0.69, 0.91), from CVD (HR 0.66; $95 \%$ CI 0.49, 0.89), but not from overall cancer (HR 0.92; $95 \%$ CI $0.75,1 \cdot 12$ ). A 2-unit increase in rMED score was associated with a $6 \%(P<0 \cdot 001)$ decreased risk of all-cause mortality. A high olive oil intake and moderate alcohol consumption contributed most to this association. In this Spanish cohort, following an olive oil-rich MD was related to a significant reduction in all-cause mortality, and reduced the risk of mortality from CVD. These results support the important role that the MD pattern has on reducing mortality in Mediterranean countries.

Key words: Mediterranean diet: Morality: EPIC-Spain: Cohort studies

The Mediterranean diet (MD) encompasses the traditional dietary patterns found in the olive-growing regions of the Mediterranean basin in the $1960 \mathrm{~s}^{(1)}$, and is globally recognised as a healthy dietary model ${ }^{(2)}$ and an intangible cultural heritage of humanity by the United Nations Educational, Scientific and Cultural Organization (UNESCO) $)^{(3)}$. The traditional

Abbreviations: EPIC, European Prospective Investigation into Cancer and Nutrition; EPIC-Spain, Spanish cohort of EPIC; HR, hazard ratio; MD, Mediterranean diet; rMED, relative Mediterranean diet.

*Corresponding author: Dr Genevieve Buckland, email gbuckland@iconcologia.net 
MD pattern is characterised by the daily use of olive oil, an abundance of plant foods such as fruit and vegetables, nuts and seeds, cereals and legumes, the consumption of fish and seafood especially in coastal regions, moderate-to-low intake of dairy products mostly from fresh cheese and yoghurt, moderate alcohol mostly in the form of wine, and a less frequent consumption of meat and meat products ${ }^{(4)}$.

Numerous epidemiological studies have explored the health benefits of the MD and evidence consistently shows that individuals who adhere to the MD have healthier ageing and a longer life $\operatorname{span}^{(5-8)}$. This is related, in part, to its role in preventing major chronic diseases such as CVD, certain cancers, type 2 diabetes and also some neurodegenerative diseases, as supported by findings from observational studies ${ }^{(6,9)}$. Furthermore, randomised controlled dietary trials have shown that the MD reduces incidence of type 2 diabetes ${ }^{(10)}$, the metabolic syndrome ${ }^{(11,12)}$ and CVD risk factors ${ }^{(13,14)}$. The MD's favourable fatty acid profile, high fibre content, wide variety of antioxidants and phytochemicals, other still unidentified biologically active compounds and their synergistic interactions can explain some of its beneficial effects on health ${ }^{(5)}$.

Over a decade ago the key elements of the MD were grouped into an a priori MD score ${ }^{(15)}$, to reflect the level of adherence to this dietary pattern. Various versions of this and other MD scores are now widely used to study the relationship between the MD pattern and different health parameters $^{(16)}$. Prospective studies have shown that following the MD is associated with a decrease in overall mortality ${ }^{(17-20)}$ and a meta-analysis of cohort studies calculated that a 2-unit increase in MD score reduced the risk of mortality by $8 \%{ }^{(9)}$. A Mediterranean-like diet has also been reported to have a beneficial effect on mortality in countries outside the Mediterranean basin ${ }^{(17,21,22)}$

Results from an elderly cohort within the European Prospective Investigation into Cancer and Nutrition (EPIC) cohort, which included subjects aged 60 years or more from nine European countries, showed that a 2-unit increase in MD score was related to a $7 \%$ reduction in mortality ${ }^{(19)}$. However, this association was not significant in six out of nine of these countries, including Spain and Italy. This may have been due to a lack of statistical power, as relatively few deaths had accrued at this time in these Mediterranean cohorts. In contrast, a previous study set in Greece observed a $14 \%$ reduction in total mortality for every 2 -unit increase in MD score ${ }^{(23)}$.

Therefore, whether the MD can reduce mortality to a similar extent in other Mediterranean populations still remains unclear. Furthermore, studies using a priori MD scores to assess the impact of the MD on mortality commonly include the MUFA:SFA ratio ${ }^{(9,16)}$ and do not directly incorporate olive oil intake, the hallmark of the MD. In previous research within the Spanish cohort of EPIC (EPIC-Spain), a high intake of fresh fruit, root vegetables and fruiting vegetables decreased the risk of mortality ${ }^{(24)}$. Therefore, we aimed to investigate the relationship between the entire MD pattern and all-cause and cause-specific mortality in the EPIC-Spain cohort.

\section{Experimental methods}

\section{Study design and population}

EPIC is a large multi-centre prospective cohort study conducted across ten European countries and designed to study the role of dietary, lifestyle, environmental and genetic factors in the development of cancer and other chronic diseases. The full methodological details have been published previously ${ }^{(25,26)}$. The present study includes participants from EPIC-Spain; 41438 healthy volunteers (15 632 men) aged 29-69 years old, recruited between 1992 and 1996 from three regions in the north (Asturias, Gipuzkoa and Navarra) and two regions in the south (Granada and Murcia). Participants were recruited from different social sectors and from both urban and rural areas (with a participation rate from 55 to $89 \%$ between centres). The majority of volunteers were active blood donors (75\%), and to a lesser extent industrial workers, civil servants or the general population. At recruitment participants gave their informed consent and the present study was approved by the Medical Ethical Committee of Bellvitge Hospital.

\section{Cohort follow-up and mortality ascertainment}

Follow-up for all-cause mortality began in 1992-93 and for this analysis was completed between December 2006 and June 2009, varying by centre. Date and underlying cause of death were obtained through record linkage with the Spanish National Statistics Institute (Instituto Nacional de Estadística; INE), which centralises this information from regional mortality registries. Cause of death was coded using International Classification of Diseases (ICD)-9 until 1999 and ICD-10 from 1999 to 2008; coding I00-I99 for CVD and C00-C99 for cancer. All other causes of death, including codes D1-D48 for in situ/benign/uncertain or unknown behaviour neoplasm (n 24), were grouped into 'other causes'. Participants with implausible dietary values, the lowest and highest $1 \%$ of the ratio of total energy intake:energy requirement, were excluded from this analysis (816 participants including fiftysix deaths). Therefore, the final study population included 40622 participants.

\section{Dietary and lifestyle data collection}

Participants were interviewed in person to collect information on usual food intake over the previous year by means of a computerised version of a validated dietary history questionnaire, used across all centres ${ }^{(27,28)}$. In a pilot study to evaluate the relative validity and reproducibility of the dietary history questionnaire, the Pearson correlation coefficients between the mean daily intake of food groups used in the relative MD (rMED) score based on the twelve $24 \mathrm{~h}$ diet recalls and the second dietary history, ranged from $0 \cdot 29$ to $0 \cdot 90^{(27)}$. Dietary information obtained from the diet history questionnaire was used to calculate ethanol intake $(\mathrm{g} / \mathrm{d})$ derived from alcoholic beverages and total energy intake $(\mathrm{kJ} / \mathrm{d})$, which was estimated using food composition tables ${ }^{(29)}$. An interviewer-administered lifestyle questionnaire was used to 
collect information on sociodemographic and lifestyle factors, including history of tobacco consumption, physical activity and reproductive indicators in women. Detailed information on medical history was collected at recruitment, including self-reported medication use and history of diseases such as diabetes, cancer and CVD. Anthropometrics (waist and hip circumference, weight and height) were measured at recruitment using standardised procedures.

\section{The relative Mediterranean diet score}

The rMED score, as previously applied in EPIC studies ${ }^{(30)}$, was used to estimate the level of adherence to the MD. It consists of an 18-point linear scale that incorporates nine selected components of the MD. Each component (apart from alcohol) is calculated as a function of energy density ( $\mathrm{g}$ per $8368 \mathrm{~kJ}$ per $\mathrm{d}(\mathrm{g}$ per $2000 \mathrm{kcal} / \mathrm{d})$ ), using the nutrient density model ${ }^{(31)}$, and then divided into tertiles of intakes. For the six components presumed to fit the MD, fruit (including nuts and seeds), vegetables (excluding potatoes), legumes, fish (fresh or frozen), olive oil and cereals, a score of 0 to 2 was assigned to the first, second and third tertile of intake. For the two components presumed to not fit the MD, total meat and dairy products, the scoring is reversed so that 2 points are assigned to the lowest tertile. Alcohol was scored dichotomously, as in previous EPIC studies ${ }^{(15)}$, assigning 2 points for moderate consumers $(5-25 \mathrm{~g} / \mathrm{d}$ for women and $10-50 \mathrm{~g} / \mathrm{d}$ for men) and 0 for participants outside these sex-specific ranges. An rMED score ranging from 0 to 18 was obtained by summing the points from each component, whereby 0 represented the lowest adherence to the MD pattern and 18 represented highest adherence. The rMED score was analysed as a continuous variable (per 2-unit increase) and as a categorical variable (low adherence, 0-6 units; medium adherence, 7-10 units; high adherence, 11-18 units).

\section{Statistical analysis}

STATA statistical software version 10 (StataCorp LP, College Station, TX, USA) was used for all analyses. The association between non-nutritional covariates and the rMED score was assessed by frequency distributions and $\chi^{2}$ tests for categorical variables and by means, standard deviations and the KruskalWallis rank sum test for continuous variables. Cox proportional hazards regression models were used to test the association between the rMED score (as a categorical and continuous variable) and all-cause mortality. Age was the primary time variable; entry time was defined as age at recruitment and exit time was defined as age at death (for cases) or last complete follow-up (for at-risk participants), depending on which occurred first. All models were stratified by centre, age at EPIC study entry and sex, and adjusted for BMI (quintiles), waist circumference (continuous), education level (none, primary, secondary, technical training, university or unspecified), validated physical activity index ${ }^{(32)}$ (inactive, moderately inactive, moderately active and active) based on a cross-tabulation of physical activity at work and during leisure-time, smoking status (never, former, current smoker of $<20$ cigarettes $/ \mathrm{d}$, current smoker of $\geq 20$ cigarettes/d and non-specified smoking status or cigarettes/d) and energy intake (continuous). Trend tests were obtained by scoring the rMED categories in a continuous scale from 1 to 3 . Two-sided $P$ values less than 0.05 were considered statistically significant.

A competing risk approach ${ }^{(33)}$ was implemented to assess the association between the MD and cause-specific mortality (CVD, cancer and other), and to test if the association between the MD and mortality differed depending on the causes of death. The Cox models were stratified by type of outcome, which allows for different associations between each risk factor and the relative hazard of each outcome, under the proportional hazard assumption. This method is based on data augmentation, as each subject needs to be assigned a separate observation for each mortality outcome. In order to compare the hazard ratios (HR) for the different causes of mortality, we first assumed different associations between the main exposure variable (the rMED score) and the covariates for each outcome, using interaction terms. This full model, with different associations between the exposure variable and covariates for each mortality outcome, was successively compared with reduced models with the same associations between these variables, using the log-likelihood ratio tests. The estimates and CI were obtained after using robust standard errors on the reduced model ${ }^{(34)}$.

Plausible effect modification by sex, age at recruitment $(<55$ years and $\geq 55$ years $)$, BMI $\left(<25,25-30, \geq 30 \mathrm{~kg} / \mathrm{m}^{2}\right)$, waist circumference (below and above the National Cholesterol Education Program-Adult Treatment Panel III cut-offs of $102 \mathrm{~cm}$ for men and $88 \mathrm{~cm}$ for women ${ }^{(35)}$ ) and smoking status (never, former, current) were explored by modelling interaction terms between each of these separate variables and the rMED score, and tested using the log-likelihood ratio test. In addition, risk of all-cause mortality in relation to categories of the rMED score was examined in analyses stratified by these cohort subgroups.

HR were calculated for each separate component of the rMED score, using the first tertile as the reference category, and mutually adjusting for the other components in the rMED score and the covariates mentioned previously. The relative importance of each of the rMED components on mortality was also assessed ${ }^{(23)}$. Each component was alternately subtracted from the original rMED score (i.e. score without fruit or without olive oil), while adjusting for the component excluded to control for potential confounding. This reduced the rMED score from $0-18$ to $0-16$, so to maintain comparability with the original range the logarithms of the HR were multiplied by 17/19 (and then expressed as a 2-unit increase) before exponentiating them.

Sensitivity analyses were carried out by additional adjustment for alcohol intake, which is associated with mortality and CHD in this cohort ${ }^{(36)}$, and for the presence of diabetes, hyperlipidaemia or hypertension. Additional models were also created excluding participants with chronic diseases at recruitment (CVD ( $n$ 9011), diabetes mellitus ( $n$ 2034) and/ or cancer $(n$ 524)), the first 2-years of follow-up (81 149 person years, including 109 cases), and mis-reporters of energy intake defined using Goldberg cut-offs ( $n 13795)^{(37)}$. 
Finally, the main Cox analyses were repeated using the original MD score constructed by Trichopoulou ${ }^{(20)}$, for ease of comparison with previous results.

\section{Results}

The cohort of 40622 subjects was followed-up for a mean of 13.4 years, during which time 1855 deaths were recorded (Table 1); 399 from CVD, 913 from cancer and 425 from other causes (118 subjects had missing information on cause of death). The mean rMED score was 8.6 (SD 2.7) overall and was highest in Granada and Murcia and lowest in Asturias.

The distribution of the baseline characteristics of the subjects according to level of adherence to the rMED is shown in Table 2. Subjects with the highest category of rMED score were older, had a higher BMI but lower energy intake $(P<0.001)$ and lower total fat and saturated fat intakes. Females, current smokers and heavy alcohol drinkers were more likely to have lower rMED scores $(P<0 \cdot 001)$.

The multivariate HR (unadjusted and fully adjusted) for the association between the rMED score and all-cause and causespecific mortality are presented in Table 3. A high compared with a low rMED score was associated with a significant 21 (95\% CI 9, 31) \% reduction in all-cause mortality and every 2-unit increase in rMED score was associated with a $6(95 \%$ CI 3, 10) \% decrease in mortality. In cause-specific mortality analyses, a high compared with a low rMED score was associated with a significant reduction in mortality from other causes (HR 0.71; 95\% CI 0.54, 0.94) and mortality from CVD (HR 0.66; $95 \%$ CI $0.49,0.89)$. A 2-unit increase in rMED score reduced the risk of CVD mortality by $16 \%(P=0.02)$ in females and by $11 \%$ in males $(P=0.01)$ (data not shown).

A high compared with a low rMED score was not significantly associated with death from overall cancer (HR 0.92; $95 \%$ CI $0 \cdot 75,1 \cdot 12)$. However, in secondary analyses including only cancers with greater evidence of being causally related to dietary factors ${ }^{(38)}$ (oesophageal, stomach, intestinal, colorectal and other digestive organ cancers, pancreatic, breast and prostate cancer) ( 570 cases), the rMED score was associated with a borderline reduction in risk of death (HR 0.79; $95 \%$ CI 0.61, $1.01 ; P=0.056$ for high $v$. low score). However, the competing risk analysis showed that there was no evidence of a significant difference in the association between the rMED score and each of the major causes of death ( $P$ for interaction $=0 \cdot 129$ ).

There was no evidence that sex modified the association between the rMED score and all-cause mortality (even though the association between the rMED score was only significant in men; Table 4), or between the rMED score and cause-specific mortality (data not shown). Hence, the main analyses are presented globally. Although the effect estimates varied in analyses stratified by age group, smoking status, BMI and waist circumference, there was no evidence of interaction with the rMED score (Table 4).

The mutually adjusted HR of mortality according to tertile of intake for the individual components of the rMED score are presented in Table 5. The majority of the Mediterranean components on their own were not significantly associated with mortality. However, a high intake of olive oil and moderate intake of alcohol were associated with a significant decreased risk of mortality, by $15 \%(P=0.006)$ and $11 \%(P=0.029)$, respectively. The negative association between moderate alcohol intake and mortality was significant in men (HR 0.87; $P=0.033$ ) but not in women (HR 0.97; $P=0.767$ ) (data not shown). As high intakes of alcohol are known to be detrimental to health, we also analysed the association between mortality and intake of alcohol separately for low intakes (less than $5 \mathrm{~g} / \mathrm{d}$ for women or $10 \mathrm{~g} / \mathrm{d}$ for men) and high intakes (more than $25 \mathrm{~g} / \mathrm{d}$ for women or $50 \mathrm{~g} / \mathrm{d}$ for men). Compared with moderate alcohol intake, high alcohol intake significantly increased risk of mortality by $17 \%(P=0.034)$, while low intake was not associated with mortality (HR 1.06; $P=0 \cdot 148$ ). We also assessed the risk of mortality for intake above $v$. below the sex-specific median of MUFA:SFA ratio (HR 1.03; $95 \%$ CI $0.93,1 \cdot 15$ ), for comparison with previous articles, even though it was not within the rMED score.

After alternately subtracting each of the nine components from the rMED score, the magnitude of the decrease in risk of mortality was generally reduced, but remained significant (Fig. 1). Fig. 1 also confirms the predominant weight that olive oil, alcohol and cereals contribute to the association between mortality and a 2-unit increase in rMED score, because alternate exclusion of these three dietary components

Table 1. Distribution of participants, all-cause and cause-specific mortality and Mediterranean diet adherence in the five centres of the Spanish cohort of the European Prospective Investigation into Cancer and Nutrition (EPIC-Spain)

\begin{tabular}{|c|c|c|c|c|c|c|c|c|c|}
\hline \multirow[b]{2}{*}{$\begin{array}{l}\text { EPIC-Spain } \\
\text { centre }\end{array}$} & \multirow[b]{2}{*}{$\begin{array}{l}\text { Cohort } \\
\text { sample }\end{array}$} & \multirow[b]{2}{*}{ Person-years } & \multicolumn{5}{|c|}{ Mortality by cause } & \multicolumn{2}{|c|}{ rMED score† } \\
\hline & & & Total $^{*}$ & $\begin{array}{l}\text { Total: } \\
\% \text { male }\end{array}$ & CVD & Cancer & Other & Mean & SD \\
\hline Asturias & 8374 & 112024 & 338 & 58 & 73 & 200 & 65 & 7.8 & 2.5 \\
\hline Granada & 7725 & 108408 & 368 & 40 & 76 & 142 & 70 & $9 \cdot 0$ & $2 \cdot 6$ \\
\hline Murcia & 8348 & 110054 & 323 & 56 & 70 & 142 & 82 & $9 \cdot 1$ & $2 \cdot 7$ \\
\hline Navarra & 7924 & 109314 & 389 & 71 & 77 & 210 & 93 & $8 \cdot 1$ & 2.7 \\
\hline Gipuzkoa & 8251 & 103266 & 437 & 73 & 103 & 219 & 115 & 8.7 & $2 \cdot 7$ \\
\hline Total & 40622 & 543066 & 1855 & 60 & 399 & 913 & 425 & $8 \cdot 6$ & $2 \cdot 7$ \\
\hline
\end{tabular}

rMED, relative Mediterranean diet.

${ }^{*}$ Cause of death is undefined for 118 cases.

† The rMED score ranges from 0 (lowest adherence) to 18 (highest adherence) and includes nine components: vegetables, legumes, fruit and nuts, cereals, fresh fish and seafood, olive oil, alcohol, meat and dairy products (from EPIC-Spain dietary questionnaire data). 
Table 2. Baseline characteristics of the 40622 participants in the Spanish cohort of the European Prospective Investigation into Cancer and Nutrition (EPIC-Spain) according to level of adherence to the relative Mediterranean diet (rMED)

\begin{tabular}{|c|c|c|c|c|c|c|c|c|}
\hline \multirow[b]{3}{*}{ Sociodemographic and lifestyle characteristics $†$} & & & \multicolumn{6}{|c|}{ rMED score* } \\
\hline & \multicolumn{2}{|c|}{$\begin{array}{l}\text { All cohort } \\
\text { (n } 40622)\end{array}$} & \multicolumn{2}{|c|}{$\begin{array}{c}\text { Low } \\
(n \text { 9294) }\end{array}$} & \multicolumn{2}{|c|}{$\begin{array}{c}\text { Medium } \\
(n 21549)\end{array}$} & \multicolumn{2}{|c|}{$\begin{array}{c}\text { High } \\
\text { (n 9779) }\end{array}$} \\
\hline & $n$ & $\%$ & $n$ & $\%$ & $n$ & $\%$ & $n$ & $\%$ \\
\hline Sex, male & 15324 & $37 \cdot 7$ & 2941 & $31 \cdot 6$ & 7946 & $36 \cdot 8$ & 4437 & $45 \cdot 0$ \\
\hline \multicolumn{9}{|l|}{ Age at enrolment (years) } \\
\hline Mean & \multicolumn{2}{|c|}{$49 \cdot 3$} & \multicolumn{2}{|c|}{48.2} & \multicolumn{2}{|c|}{$49 \cdot 3$} & \multicolumn{2}{|c|}{$50 \cdot 2$} \\
\hline SD & \multicolumn{2}{|c|}{$8 \cdot 0$} & \multicolumn{2}{|c|}{$8 \cdot 0$} & \multicolumn{2}{|c|}{$8 \cdot 0$} & & \\
\hline BMI $\left(\mathrm{kg} / \mathrm{m}^{2}\right)$ & & & & & & & & \\
\hline Mean & 28 & & & & 28 & & & \\
\hline SD & 4. & & & & 4 & & & \\
\hline Waist circumference $\neq$ & & & & & & & & \\
\hline Men $<102 \mathrm{~cm}$, women $<88 \mathrm{~cm}$ & 23400 & $57 \cdot 6$ & 5446 & $58 \cdot 6$ & 12365 & 57.4 & 5589 & $57 \cdot 1$ \\
\hline Men $\geq 102 \mathrm{~cm}$, women $\geq 88 \mathrm{~cm}$ & 17222 & $42 \cdot 4$ & 3848 & 41.4 & 9184 & $42 \cdot 6$ & 4190 & $42 \cdot 9$ \\
\hline Educational level & & & & & & & & \\
\hline No formal education & 12372 & 30.5 & 2700 & $29 \cdot 1$ & 6640 & $30 \cdot 8$ & 3032 & $31 \cdot 0$ \\
\hline Primary school & 15687 & $38 \cdot 6$ & 3871 & $41 \cdot 7$ & 8321 & 38.6 & 3495 & 35.7 \\
\hline Secondary school & 2628 & $6 \cdot 5$ & 657 & $7 \cdot 1$ & 1365 & $6 \cdot 3$ & 606 & $6 \cdot 2$ \\
\hline Technical or professional training & 3347 & $8 \cdot 2$ & 732 & 7.9 & 1749 & $8 \cdot 1$ & 866 & 8.9 \\
\hline University degree & 4677 & 11.5 & 981 & $10 \cdot 6$ & 2501 & $11 \cdot 6$ & 1195 & $12 \cdot 2$ \\
\hline Not specified & 1911 & 4.7 & 353 & $3 \cdot 8$ & 973 & 4.5 & 585 & $6 \cdot 0$ \\
\hline Physical activity & & & & & & & & \\
\hline Active & 4363 & $10 \cdot 7$ & 876 & $9 \cdot 4$ & 2277 & $10 \cdot 6$ & 1210 & $12 \cdot 4$ \\
\hline Moderately active & 7133 & $17 \cdot 6$ & 1504 & $16 \cdot 2$ & 3846 & $17 \cdot 9$ & 1783 & $18 \cdot 2$ \\
\hline Moderately inactive & 13509 & 33.3 & 3257 & $35 \cdot 0$ & 7138 & 33.1 & 3114 & 31.8 \\
\hline Inactive & 15617 & 38.4 & 3657 & 39.4 & 8288 & 38.5 & 3672 & 37.6 \\
\hline Smoking status & & & & & & & & \\
\hline Never & 22538 & 55.5 & 5121 & $55 \cdot 1$ & 12160 & $56 \cdot 4$ & 5257 & 53.8 \\
\hline Former & 7183 & $17 \cdot 7$ & 1368 & $14 \cdot 7$ & 3730 & $17 \cdot 3$ & 2085 & $21 \cdot 3$ \\
\hline Current ( $<20$ cigarettes/d) & 5541 & $13 \cdot 6$ & 1335 & 14.4 & 2920 & $13 \cdot 6$ & 1286 & $13 \cdot 2$ \\
\hline Current ( $\geq 20$ cigarettes $/ d$ ) & 3838 & 9.5 & 1141 & $12 \cdot 3$ & 1941 & $9 \cdot 0$ & 756 & $7 \cdot 7$ \\
\hline Not specified§ & 1522 & $3 \cdot 8$ & 329 & 3.7 & 798 & 3.7 & 395 & 4.0 \\
\hline Alcohol intake & & & & & & & & \\
\hline $0 \mathrm{~g} / \mathrm{d}$ & 15303 & $37 \cdot 7$ & 4185 & $45 \cdot 0$ & 8385 & 38.9 & 2733 & 28.0 \\
\hline$<10 \mathrm{~g} / \mathrm{d}$ & 10686 & $26 \cdot 3$ & 2502 & $26 \cdot 9$ & 5735 & $26 \cdot 6$ & 2449 & $25 \cdot 0$ \\
\hline$\geq 10$ and $<20 \mathrm{~g} / \mathrm{d}$ & 4374 & $10 \cdot 8$ & 395 & $4 \cdot 3$ & 2060 & $9 \cdot 6$ & 1919 & 19.6 \\
\hline$\geq 20$ and $<40 \mathrm{~g} / \mathrm{d}$ & 4806 & $11 \cdot 8$ & 648 & $7 \cdot 0$ & 2407 & $11 \cdot 2$ & 1751 & 17.9 \\
\hline$\geq 40 \mathrm{~g} / \mathrm{d}$ & 5453 & 13.4 & 1564 & $16 \cdot 8$ & 2962 & 13.7 & 927 & 9.5 \\
\hline Energy intake (kJ/d) & & & & & & & & \\
\hline Mean & 89 & & & & 88 & & & \\
\hline SD & 278 & & & & 279 & & & \\
\hline Carbohydrate intake (g/4184 kJ per d) & & & & & & & & \\
\hline Median & 103 & & & & 10 & & & \\
\hline SD & 17 & & & & 17 & & & \\
\hline Protein intake (g/4184 kJ per d) & & & & & & & & \\
\hline Median & 47 & & & & 48 & & & \\
\hline SD & 7 . & & & & 7 & & & \\
\hline Total fat intake (g/4184 kJ per d) & & & & & & & & \\
\hline Median & 39 & & & & 39 & & & \\
\hline SD & 6 . & & & & 6 & & & \\
\hline MUFA intake ( $g / 4184 \mathrm{~kJ}$ per $\mathrm{d})$ & & & & & & & & \\
\hline Median & 17 & & & & 17 & & & \\
\hline SD & 4. & & & & 4 & & & \\
\hline SFA intake (g/4184 kJ per d) & & & & & & & & \\
\hline Median & 12 & & & & 12 & & & \\
\hline SD & 3. & & & & 3 & & & \\
\hline PUFA intake (g/4184 kJ per d) & & & & & & & & \\
\hline Median & 5 . & & & & 5 & & & \\
\hline SD & 2 . & & & & 2 & & & \\
\hline MUFA:SFA ratio & & & & & & & & \\
\hline Median & 1. & & & & 1 & & & \\
\hline SD & 0.4 & & & & 0 & & & \\
\hline
\end{tabular}

* rMED low score, 0-6; medium score, 7-10; high score, 11-18.

† All comparisons between rMED groups and tests for trend were statistically significant $\left(P<0.001 ; \chi^{2}\right.$ for categorical variables or the Kruskal-Wallis rank sum test for continuous variables), except for waist circumference $(P=0.082)$.

‡ National Cholesterol Education Program's Adult Treatment Panel III cut-offs.

$\S$ Not specified refers to subjects with missing information on smoking status or number of cigarettes smoked per $\mathrm{d}$. 
Table 3. Association between adherence to the relative Mediterranean diet (rMED) and all-cause and cause-specific mortality in the Spanish cohort of the European Prospective Investigation into Cancer and Nutrition (EPIC-Spain)

(Hazard ratios (HR) and $95 \%$ confidence intervals)

\begin{tabular}{|c|c|c|c|c|c|c|c|c|c|c|}
\hline \multirow[b]{3}{*}{ Mortality cause $\dagger$} & \multicolumn{7}{|c|}{ rMED score: categorical ${ }^{*}$} & \multicolumn{3}{|c|}{ rMED score: continuous } \\
\hline & \multicolumn{2}{|c|}{ Low ( $n$ 9294) } & \multicolumn{2}{|c|}{ Medium ( $n 21549$ ) } & \multicolumn{2}{|c|}{ High ( $n$ 9779) } & \multirow[b]{2}{*}{$P$ for trend } & \multicolumn{2}{|c|}{ 2-Unit increase } & \multirow[b]{2}{*}{$P$} \\
\hline & $\mathrm{HR}$ & $95 \% \mathrm{Cl}$ & HR & $95 \% \mathrm{Cl}$ & $\mathrm{HR}$ & $95 \% \mathrm{Cl}$ & & $\mathrm{HR}$ & $95 \% \mathrm{Cl}$ & \\
\hline All-cause deaths $(n)$ & & 431 & & 967 & & 457 & & & 1855 & \\
\hline Unadjusted HR $\ddagger$ & 1 & Reference & 0.84 & $0.75,0.95$ & 0.74 & $0.65,0.85$ & $<0.001$ & 0.92 & $0.89,0.95$ & $<0.001$ \\
\hline Multivariate HR§ & 1 & Reference & 0.88 & $0.79,0.99$ & 0.79 & $0.69,0.91$ & 0.001 & 0.94 & $0.90,0.97$ & $<0.001$ \\
\hline CVD deaths $(n)$ & & 92 & & 13 & & 94 & & & 399 & \\
\hline Unadjusted HR $\ddagger$ & 1 & Reference & 0.84 & $0.65,1.08$ & 0.67 & $0.50,0.90$ & 0.007 & 0.88 & $0.82,0.95$ & 0.001 \\
\hline Multivariate HR§ & 1 & Reference & 0.84 & $0.66,1.09$ & 0.66 & $0.49,0.89$ & 0.006 & 0.88 & $0.81,0.95$ & $<0.001$ \\
\hline Cancer deaths $(n)$ & & 204 & & 186 & & 223 & & & 913 & \\
\hline Unadjusted HR $\ddagger$ & 1 & Reference & 0.95 & $0.80,1.12$ & 0.85 & $0.70,1.04$ & 0.108 & 0.96 & $0.92,1.01$ & 0.135 \\
\hline Multivariate HR§ & 1 & Reference & 0.99 & $0.84,1.18$ & 0.92 & $0.75,1.12$ & 0.414 & 0.98 & $0.93,1.03$ & 0.510 \\
\hline Other deaths $(n)$ & & 110 & & 207 & & 108 & & & 425 & \\
\hline Unadjusted HR & 1 & Reference & 0.68 & $0.54,0.87$ & 0.66 & $0.50,0.86$ & 0.004 & 0.88 & $0.82,0.95$ & 0.001 \\
\hline Multivariate HR§ & 1 & Reference & 0.72 & $0.57,0.91$ & 0.71 & $0.54,0.94$ & 0.022 & 0.90 & $0.84,0.97$ & 0.008 \\
\hline
\end{tabular}

* rMED low score, 0-6; medium score, 7-10; high score, 11-18.

† Cause-specific analysis excludes 118 cases with no information on cause of death.

$\ddagger$ Cox proportional hazards regression analysis stratified by centre, age and sex.

$\S$ Adjusted for BMI, waist circumference, education level, physical activity, smoking status and intensity and total energy intake.

from the score resulted in the greatest percentage reduction in effect $(-27 \cdot 7,-18.5$ and $-15 \cdot 4 \%$, respectively).

The negative association between mortality and the rMED score was similar in the sensitivity analyses (Table 6), where additional adjustments were made for alcohol intake and the presence of diabetes, hypertension or hyperlipidaemia or a history of CVD, cancer or diabetes at recruitment or after the exclusion of the first 2 years of follow up and mis-reporters of energy intake. The exclusion of subjects with chronic diseases at recruitment (cancer, CVD or diabetes) resulted in a minimal attenuation of the association (HR 0.95; 95\% CI 0.91, 1.00; for a 2-unit increase in rMED score).

Table 4. Association between adherence to the relative Mediterranean diet (rMED) and mortality in the population subgroups in the Spanish cohort of the European Prospective Investigation into Cancer and Nutrition (EPIC-Spain)

(Hazard ratios (HR) and $95 \%$ confidence intervals)

\begin{tabular}{|c|c|c|c|c|c|c|c|c|c|c|}
\hline \multirow[b]{3}{*}{ Cohort subgroup } & \multirow[b]{3}{*}{ Cases $(n)$} & \multirow[b]{3}{*}{ Person-years } & \multicolumn{6}{|c|}{ rMED score: categorical* } & \multirow{3}{*}{$\begin{array}{l}P \text { for } \\
\text { trend }\end{array}$} & \multirow{3}{*}{$\begin{array}{c}P \text { for } \\
\text { interaction }\end{array}$} \\
\hline & & & \multicolumn{2}{|r|}{ Low } & \multicolumn{2}{|c|}{ Medium } & \multicolumn{2}{|c|}{ High } & & \\
\hline & & & $\mathrm{HR} \dagger$ & $95 \% \mathrm{Cl}$ & $\mathrm{HR} \dagger$ & $95 \% \mathrm{Cl}$ & $\mathrm{HR} \dagger$ & $95 \% \mathrm{Cl}$ & & \\
\hline \multicolumn{11}{|l|}{ Sex } \\
\hline Male & 1121 & 203038 & 1 & Reference & 0.88 & $0.75,1.02$ & $0 \cdot 76$ & $0.63,0.90$ & 0.002 & \\
\hline Female & 734 & 340028 & 1 & Reference & 0.89 & $0.75,1.06$ & 0.85 & $0.68,1.06$ & $0 \cdot 152$ & 0.512 \\
\hline \multicolumn{11}{|l|}{ Age at recruitment } \\
\hline$<55$ years & 819 & 399653 & 1 & Reference & 0.95 & $0.80,1 \cdot 12$ & 0.93 & $0 \cdot 76,1 \cdot 14$ & 0.492 & \\
\hline$>55$ years & 1036 & 143413 & 1 & Reference & 0.83 & $0.71,0.97$ & $0 \cdot 70$ & $0.58,0.84$ & $<0.001$ & 0.312 \\
\hline \multicolumn{11}{|l|}{ Smoking status $\ddagger$} \\
\hline Never & 791 & 302650 & 1 & Reference & 0.85 & $0.71,1.01$ & 0.73 & $0.59,0.91$ & 0.005 & \\
\hline Former & 382 & 95893 & 1 & Reference & 0.93 & $0.69,1.23$ & 0.89 & $0.64,1.22$ & 0.468 & \\
\hline Current & 682 & 144217 & 1 & Reference & 0.94 & $0 \cdot 78,1 \cdot 14$ & 0.83 & $0.66,1.04$ & $0 \cdot 113$ & 0.775 \\
\hline \multicolumn{11}{|l|}{ BMI } \\
\hline$<25 \mathrm{~kg} / \mathrm{m}^{2}$ & 307 & 118872 & 1 & Reference & 0.90 & $0.67,1.20$ & 0.87 & $0.60,1.25$ & 0.435 & \\
\hline $25-29 \mathrm{~kg} / \mathrm{m}^{2}$ & 852 & 260963 & 1 & Reference & 0.81 & $0.68,0.96$ & 0.77 & $0.63,0.94$ & 0.016 & \\
\hline$\geq 30 \mathrm{~kg} / \mathrm{m}^{2}$ & 696 & 163231 & 1 & Reference & 0.94 & $0.78,1.14$ & 0.79 & $0.63,1.00$ & 0.048 & 0.455 \\
\hline \multicolumn{11}{|l|}{ Waist circumference§ } \\
\hline $\begin{array}{l}\text { Men }<102 \mathrm{~cm} \\
\text { women }<88 \mathrm{~cm}\end{array}$ & 900 & 312902 & 1 & Reference & 0.78 & $0.66,0.93$ & $0 \cdot 78$ & $0.65,0.95$ & 0.018 & \\
\hline $\begin{array}{l}\text { Men } \geq 102 \mathrm{~cm} \\
\text { women } \geq 88 \mathrm{~cm}\end{array}$ & 955 & 230164 & 1 & Reference & 0.99 & $0 \cdot 84,1 \cdot 17$ & $0 \cdot 81$ & $0.66,0.99$ & 0.032 & 0.062 \\
\hline
\end{tabular}

* rMED low score, 0-6; medium score, 7-10; high score, 11-18.

† Cox proportional hazards regression analysis: stratified by centre, age and sex and adjusted for BMI, waist circumference, education level, physical activity, smoking status and intensity and total energy intake.

$\ddagger$ Stratified models of smoking status exclude twenty-two non-cases with unknown smoking status. Former smokers additionally adjusted for duration of smoking and current smokers additionally adjusted for number of cigarettes smoked per $\mathrm{d}$.

$\S$ Waist circumference cut-offs correspond to the National Cholesterol Education Program's Adult Treatment Panel III criteria. 
Table 5. Mortality risk according to tertile intake of each component of the relative Mediterranean diet (rMED) in the Spanish cohort of the European Prospective Investigation into Cancer and Nutrition (EPIC-Spain) (Mutually adjusted hazard ratios (HR) and $95 \%$ confidence intervals)

\begin{tabular}{|c|c|c|c|c|c|}
\hline \multirow[b]{2}{*}{$\begin{array}{l}\text { rMED score } \\
\text { component }\end{array}$} & \multirow[b]{2}{*}{$\begin{array}{l}\text { Tertile }(\mathrm{g} / 8368 \mathrm{~kJ} \\
(2000 \mathrm{kcal}) \text { per d) }\end{array}$} & \multicolumn{4}{|c|}{ Total ( $n 40622$ ) } \\
\hline & & $\begin{array}{l}\text { Cases } \\
(n 1855)\end{array}$ & $\mathrm{HR}^{*}$ & $95 \% \mathrm{Cl}$ & $\begin{array}{l}P \text { for } \\
\text { trend }\end{array}$ \\
\hline \multirow[t]{3}{*}{ Vegetables } & $<164 \cdot 3$ & 687 & 1 & Reference & \\
\hline & $164 \cdot 3$ to $<282 \cdot 2$ & 592 & 0.95 & $0.84,1.06$ & \\
\hline & $\geq 282.2$ & 576 & 1.02 & $0.90,1.16$ & 0.736 \\
\hline \multirow[t]{3}{*}{ Fruit and nuts } & $<204.4$ & 695 & 1 & Reference & \\
\hline & 204.4 to $<380.0$ & 584 & 0.91 & $0.81,1.02$ & \\
\hline & $\geq 380.0$ & 576 & 0.93 & $0.82,1.06$ & 0.250 \\
\hline \multirow[t]{3}{*}{ Legumes } & $<32 \cdot 1$ & 611 & 1 & Reference & \\
\hline & 32.1 to $<56.0$ & 548 & 0.92 & $0.82,1.03$ & \\
\hline & $\geq 56.0$ & 696 & 1.00 & $0.89,1.12$ & 0.972 \\
\hline \multirow[t]{3}{*}{ Fish and seafood } & $<35.2$ & 562 & 1 & Reference & \\
\hline & 35.2 to $<64.1$ & 634 & $1 \cdot 13$ & $1 \cdot 00,1 \cdot 27$ & \\
\hline & $\geq 64.1$ & 659 & 1.02 & $0.90,1.15$ & 0.896 \\
\hline \multirow[t]{3}{*}{ Cereals } & $<151.8$ & 614 & 1 & Reference & \\
\hline & 151.8 to $<214.0$ & 604 & 0.92 & $0.82,1.03$ & \\
\hline & $\geq 214.0$ & 637 & 0.91 & $0.81,1.03$ & 0.132 \\
\hline \multirow[t]{3}{*}{ Olive oil } & $<13.7$ & 710 & 1 & Reference & \\
\hline & 13.7 to $<24.7$ & 578 & 0.93 & $0.83,1.04$ & \\
\hline & $\geq 24.7$ & 567 & 0.85 & $0.75,0.96$ & 0.006 \\
\hline \multirow[t]{2}{*}{ Alcohol† } & Outside range & 1287 & 1 & Reference & \\
\hline & Inside range & 568 & 0.89 & $0.80,0.99$ & 0.029 \\
\hline \multirow[t]{3}{*}{ Dairy } & $<198.5$ & 712 & 1 & Reference & \\
\hline & 198.5 to $<352.0$ & 568 & 1.02 & $0.90,1.14$ & \\
\hline & $\geq 352.0$ & 575 & 1.08 & $0.95,1.24$ & 0.176 \\
\hline \multirow[t]{3}{*}{ Meat } & $<96.9$ & 625 & 1 & Reference & \\
\hline & 96.9 to $<136.6$ & 574 & 0.95 & $0.85,1.07$ & \\
\hline & $\geq 136 \cdot 6$ & 656 & 1.08 & $0.96,1.22$ & 0.233 \\
\hline
\end{tabular}

* Cox proportional hazards regression analysis: stratified by centre, age and sex and adjusted for BMI, waist circumference, education level, physical activity, smoking status and intensity, total energy intake and mutually adjusted for all rMED score components in the Table.

† Alcohol expressed as g/ethanol per d, not as a function of energy density: Inside range for males: $\geq 10 \mathrm{~g}$ to $<50 \mathrm{~g}$, and for females: $\geq 5 \mathrm{~g}$ to $<25 \mathrm{~g}$.

Finally, we repeated the analysis with the original MD score developed by Trichopoulou et al. ${ }^{(20)}$ and found that a high $v$. a low MD score was associated with a $17(95 \%$ CI 5, 27) \% reduction in mortality.

\section{Discussion}

The role of a Mediterranean dietary pattern on all-cause and cause-specific mortality was assessed within the Spanish adult population. The present results show that high adherence to the MD was related to a significant $21 \%$ reduction in all-cause mortality and a $34 \%$ reduction in CVD. The beneficial effect of the MD on mortality seemed to be predominantly due to the abundance of olive oil and moderate alcohol intake within this dietary pattern.

The present results are in line with previous findings, where high adherence to a MD was associated with a reduction in mortality by $21 \%$ in men and by $20 \%$ in women in the $\mathrm{USA}^{(17)}$, and by $17 \%{ }^{(19)}$ and $25 \%{ }^{(39)}$ in two elderly European cohorts. Findings from prospective studies in Sweden ${ }^{(21)}$, Denmark $^{(40)}$ and even Australia ${ }^{(41)}$ also support the protective effect of a Mediterranean-like diet on risk of dying. Nevertheless, it is also of interest to explore this relationship within exclusively Mediterranean populations, as this is where the closest conformity to a genuine MD is found (including a habitual and high intake of olive oil), and where the greatest benefits would be presumed to be seen. To date this had only been studied in the Greek population ${ }^{(23)}$, where a 2-unit increase in a 9-unit MD score was associated with a $14 \%$ decreased risk of dying. This was comparable with the association observed in our Spanish population, where an increase in the rMED score of a similar proportion (4-unit increase due to a 18 -unit scale) was associated with a $12 \%$ decreased risk of dying. However, direct comparison of results between studies should be made with caution, considering that the variations in the magnitude of the association are likely to be influenced by many factors besides the study population. One such factor is variation in the MD score due to inclusion of different MD components (i.e. MUFA:SFA ratio instead of olive oil) or the use of energy density of intakes and tertiles as cut-offs instead of sex-specific medians.

In cause-specific mortality analyses it was clear that the MD was beneficial for mortality from CVD. This finding has been reported by several other studies ${ }^{(9)}$ and the cardioprotective role of the MD is now well documented ${ }^{(9,42)}$ and mechanisms that mediate this effect are increasingly understood ${ }^{(12,13,42,43)}$ In previous studies on the MD and cause-specific mortality, the greatest benefit of the MD was consistently seen in mortality from $\operatorname{CVD}^{(17,20,39)}$, in particular from $\mathrm{CHD}^{(20,39)}$. 


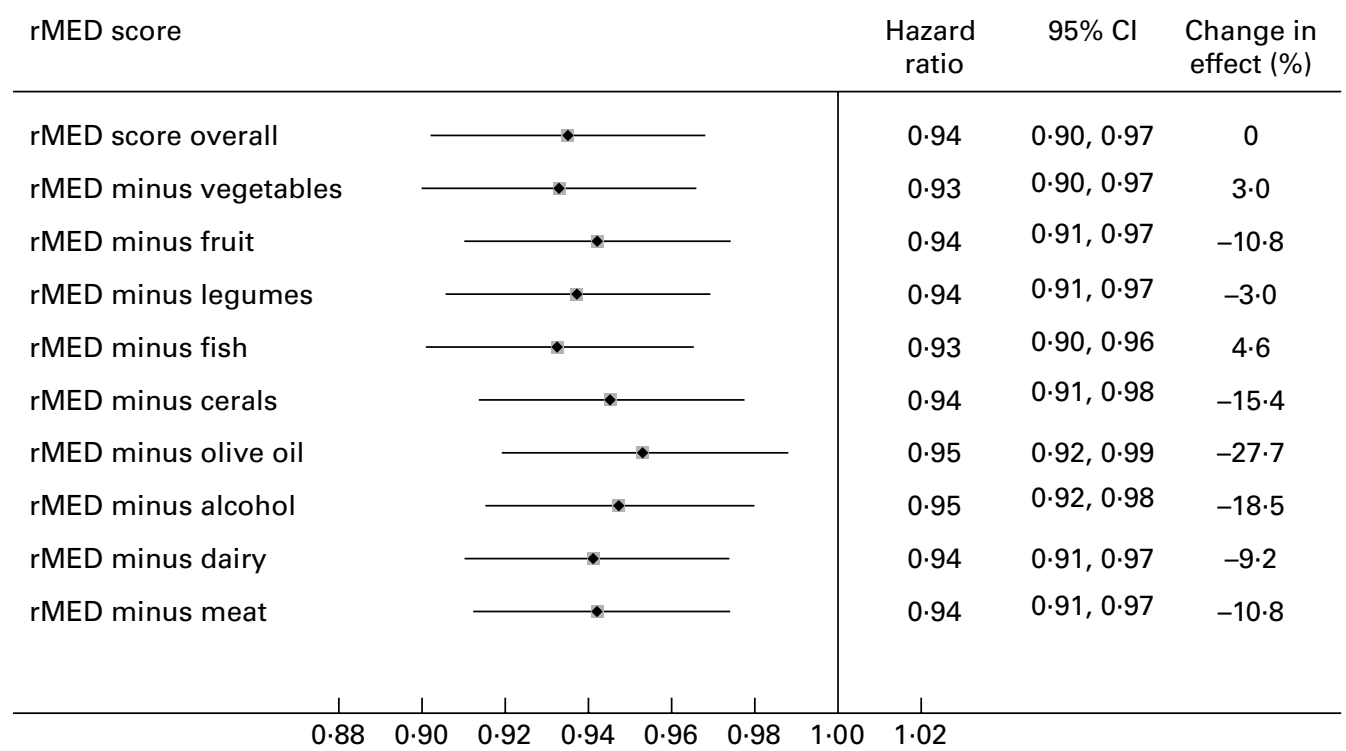

Fig. 1. Association between mortality and a 2-unit increase in the relative Mediterranean diet (rMED) score while alternately subtracting each component.

It is also worth noting that there was an important negative association for death from other causes, which has also been reported previously ${ }^{(17,39)}$. In the present study this category includes death from other types of chronic diseases, respiratory disorders and other diseases and infections, as well as external causes. It is likely that a broad spectrum of nutritional attributes of the $\mathrm{MD}^{(2,5,7,44)}$, such as its rich source of varied antioxidants, favourable lipid profile, high fibre content and abundance of phytochemicals, could help prevent or improve the prognosis or response to treatment of a wide range of illnesses ${ }^{(6,45)}$, thereby reducing mortality.

The MD did not appear to reduce mortality from cancer when all cancers were analysed together, a finding common to other studies ${ }^{(21,39)}$, although not all ${ }^{(17,20)}$. This is not unexpected considering cancer risk factors vary greatly depending on the cancer site ${ }^{(38)}$, and cancer is often described as a constellation of many different diseases, some much less dietary related. Along these lines, we found that there was a stronger borderline significant association between the MD and the more dietary related cancers. This is consistent with evidence from previous studies that have observed that the MD is related to a reduced risk of stomach, colorectal, breast and upper aerodigestive tract cancers ${ }^{(46)}$.

The protective effect of the MD on mortality appeared to be predominantly driven by high olive oil and moderate alcohol intake. In terms of olive oil, there was a notable $15 \%$ decreased mortality for subjects whose consumption of olive oil was in the highest tertile. Although a previous study in EPIC-Greece found that an increment of $20 \mathrm{~g}$ olive oil per $\mathrm{d}$ did not significantly reduce mortality, regular olive oil consumption has been shown to decrease mortality in survivors of a myocardial infarction ${ }^{(47)}$ and reduce the likelihood of having a first acute myocardial infarction ${ }^{(48)}$. Olive oil is particularly renowned for its cardioprotective properties ${ }^{(44,49)}$, in line with results from EPIC-Spain where the top tertile of olive oil intake was related to a $18 \%$ reduction in CHD incidence ${ }^{(30)}$. In addition, the Italian EPICOR Study (Italian cardiovascular section of EPIC) found that the top quartile of olive oil consumption was associated with a $44 \%$ reduction in CHD risk in women ${ }^{(50)}$.

Table 6. Sensitivity analyses for the association between mortality and each 2-unit increase in the relative Mediterranean diet (rMED) in the Spanish cohort of the European Prospective Investigation into Cancer and Nutrition (EPIC-Spain)

(Hazard ratios (HR) and $95 \%$ confidence intervals)

\begin{tabular}{|c|c|c|c|c|c|}
\hline \multirow[b]{3}{*}{ Characteristics } & \multirow[b]{3}{*}{ Cases $(n)$} & \multirow[b]{3}{*}{ At risk $(n)$} & \multicolumn{3}{|c|}{ rMED score*: continuous } \\
\hline & & & \multicolumn{2}{|c|}{ 2-Unit increase } & \multirow[b]{2}{*}{$P$} \\
\hline & & & $\mathrm{HR}$ & $95 \% \mathrm{Cl}$ & \\
\hline \multicolumn{6}{|l|}{ Additional adjusting by: } \\
\hline Alcohol intake† & 1855 & 38767 & 0.94 & $0.91,0.98$ & 0.001 \\
\hline Diabetes, hypertension and hyperlipidaemia or medication for such disorders & 1855 & 38767 & 0.93 & $0.90,0.97$ & $<0.001$ \\
\hline \multicolumn{6}{|l|}{ Excluding: } \\
\hline First 2 years of follow-up & 1746 & 38767 & 0.93 & 0.90 .0 .97 & $<0.001$ \\
\hline Subjects with chronic diseases at baseline (cancer, CVD, diabetes) & 1068 & 29060 & 0.95 & $0.91,1.00$ & 0.035 \\
\hline Mis-reporters of energy according to Goldberg classification ${ }^{(37)}$ & 1206 & 25621 & 0.94 & $0.90,0.98$ & 0.007 \\
\hline
\end{tabular}


Moderate alcohol consumption also contributed considerably to the protective effect of the MD pattern on mortality, and when its effect was analysed separately a moderate compared with a low or high alcohol intake was associated with an $11 \%$ decreased risk of dying. Moderate alcohol intake is associated with a decreased risk of mortality, mainly from $\mathrm{CHD}^{(51)}$. Accordingly, alcohol was inversely associated with the incidence of CHD in a previous study within this cohort $^{(36)}$, and a study assessing the relative importance of each of the MD components on total mortality in Greece showed that moderate alcohol consumption contributed most within the MD score ${ }^{(23)}$. In secondary analyses we found that high alcohol intake significantly increased risk of mortality compared with moderate intake, which is in line with evidence showing that high alcohol intake is detrimental to health, and is a risk factor for some cancers ${ }^{(38)}$. However, a low intake of alcohol was not associated with mortality. Nevertheless, the low intake group can be difficult to interpret, as it contains never drinkers, low drinkers and also heavy drinkers who have stopped drinking for health reasons. Despite this, a possible limitation of the rMED score is that equal weight is given to low and high alcohol intake (assigning 0 points to each), even though their effect on health is distinct ${ }^{(51)}$. In future analyses it may be more appropriate to differentiate the scoring of abstainers or low alcohol drinkers from heavy drinkers.

In the present study the highest $v$. the lowest tertile of intake of fruit and vegetables was not associated with mortality. However, in a previous study in this cohort $^{(24)}$, the highest $v$. the lowest quartile of fresh fruit, root vegetables and fruiting vegetables were all associated with a significant reduction in overall mortality, suggesting that a significant protective effect for some components might only be seen when comparing more extreme levels of intake or subgroups of these food groups.

Although it is useful to understand the relative importance of each of the components of the MD and the possible biological mechanisms involved, it is important to highlight that no one component was wholly responsible for the observed association. The overall Mediterranean dietary pattern conferred the greatest benefit, as this combines the health effects of many food groups and the biological interactions that take place between them ${ }^{(16)}$. This is also one of the advantages of using dietary indices such as the rMED score to study the effect of this dietary pattern on health outcomes. Other strengths of the present study include its prospective design with a long follow-up period and sufficient number of deaths to enable stratification by cause of death and by cohort subgroups. In addition, a large number of plausible confounders and effect modifiers were taken into account in the analyses.

There are some study limitations that deserve discussion. Reverse causality may exist, as subjects with CVD or cancer or with early symptoms of these diseases may have already altered their diet or lifestyle as a consequence. However, adjusting for the presence of diabetes, hypertension or hyperlipidaemia or excluding cases diagnosed during the first 2 years of follow-up or subjects with CVD, cancer or diabetes at baseline, did not notably alter the association. Another limitation is from dietary measurement error; however, a validated interviewer-administered diet history questionnaire was used, which has fewer measurement errors than $\mathrm{FFQ}^{(52)}$, and also covered over 600 food and recipe items ${ }^{(27,28)}$. Reporting bias is a potential limitation, and is especially prevalent in obese individuals who commonly report implausibly low energy intakes $^{(53)}$. Therefore, we applied more stringent criteria using Goldberg cut-offs ${ }^{(37)}$ to identify subjects with implausible dietary intakes. However, when these subjects were excluded in sensitivity analyses the association between the MD and mortality did not change. Since close adherence to the MD was linked to a generally healthier lifestyle (i.e. doing more physical activity and giving up smoking) and also to a higher education level, there could be residual confounding from lifestyle characteristics or certain medication use (i.e. aspirin) that was not ascertained, or from variables adjusted for but measured imprecisely. However, since the key known confounders were taken into account, we assume this could only have a minimal impact on the results.

This large prospective study supports the important role that an olive oil-rich MD plays on reducing mortality in Mediterranean populations, and the extent of the beneficial effect is in line with previous research in Mediterranean countries. The present results also confirm that the MD is associated with a significant and substantial reduction in CVD mortality and has beneficial effects on selected cancers that are potentially more diet related. These health benefits underscore the MD's importance as a cultural heritage of humanity.

\section{Acknowledgements}

The present study was supported by the European Commission (DG-SANCO), the International Agency for Research on Cancer (IARC), the Health Research Funds (FIS) of the Spanish Ministry of Health (grant no. Exp P10710130) from the 'Instituto de Salud Carlos III'; the Spanish Regional Governments of Andalucía, Asturias, Basque Country, Murcia (no. 6236) and Navarra and the Catalan Institute of Oncology, and Red Temática de Investigación Cooperativa en Cáncer (RTICC) (grant no. C03/10, R06/0020).

All authors have contributed towards the different phases of manuscript preparation (the study design, interpretation of the results and drafting of the manuscript or revising it critically for important intellectual content) and also approved the final submitted version. In particular, G. B. was responsible for the statistical analysis and N. T. for the competing risk analysis. G. B. was also responsible for drafting the manuscript and C. A. G. is the guarantor.

All authors declare that there are no conflicts of interest in relation to this article.

\section{References}

1. Trichopoulou A \& Lagiou P (1997) Healthy traditional Mediterranean diet: an expression of culture, history, and lifestyle. Nutr Rev 55, 383-389. 
2. Willett WC, Sacks F, Trichopoulou A, et al. (1995) Mediterranean diet pyramid: a cultural model for healthy eating. $A m J$ Clin Nutr 61, S1402-S1406.

3. United Nations Educational, Scientific and Cultural Organization (2011) Representative List of the Intangible Cultural Heritage of Humanity http://www.unesco.org/culture/ich/ en/RL/00394 (accessed December 2010).

4. Serra-Majem L, Trichopoulou A, Ngo de la Cruz J, et al. (2004) Does the definition of the Mediterranean diet need to be updated? Public Health Nutr 7, 927-929.

5. Perez-Lopez FR, Chedraui P, Haya J, et al. (2009) Effects of the Mediterranean diet on longevity and age-related morbid conditions. Maturitas 64, 67-79.

6. Roman B, Carta L, Martinez-Gonzalez MA, et al. (2008) Effectiveness of the Mediterranean diet in the elderly. Clin Interv Aging 3, 97-109.

7. Trichopoulou A \& Vasilopoulou E (2000) Mediterranean diet and longevity. Br J Nutr 84, Suppl. 2, S205-S209.

8. Keys A, Menotti A, Karvonen MJ, et al. (1986) The diet and 15-year death rate in the seven countries study. Am J Epidemiol 124, 903-915.

9. Sofi F, Abbate R, Gensini GF, et al. (2010) Accruing evidence about benefits of adherence to the Mediterranean diet on health: an updated systematic review and meta-analysis. Am J Clin Nutr 92, 1189-1196.

10. Salas-Salvado J, Bullo M, Babio N, et al. (2010) Reduction in the incidence of type 2-diabetes with the Mediterranean diet: results of the PREDIMED-Reus Nutrition Intervention Randomized Trial. Diabetes Care 34, 14-19.

11. Salas-Salvado J, Fernandez-Ballart J, Ros E, et al. (2008) Effect of a Mediterranean diet supplemented with nuts on metabolic syndrome status: one-year results of the PREDIMED randomized trial. Arch Intern Med 168, 2449-2458.

12. Esposito K, Marfella R, Ciotola M, et al. (2004) Effect of a Mediterranean-style diet on endothelial dysfunction and markers of vascular inflammation in the metabolic syndrome: a randomized trial. JAMA 292, 1440-1446.

13. Estruch R, Martínez-González MA, Corella D, et al. (2006) Effects of a Mediterranean-style diet on cardiovascular risk factors: a randomized trial. Ann Intern Med 145, 1-11.

14. Estruch R (2010) Anti-inflammatory effects of the Mediterranean diet: the experience of the PREDIMED study. Proc Nutr Soc 69, 333-340.

15. Trichopoulou A, Kouris-Blazos A, Wahlqvist ML, et al. (1995) Diet and overall survival in elderly people. BMJ 311, 1457-1460.

16. Bach A, Serra-Majem L, Carrasco JL, et al. (2006) The use of indexes evaluating the adherence to the Mediterranean diet in epidemiological studies: a review. Public Health Nutr $\mathbf{9}$, $132-146$

17. Mitrou PN, Kipnis V, Thiebaut AC, et al. (2007) Mediterranean dietary pattern and prediction of all-cause mortality in a US population: results from the NIH-AARP Diet and Health Study. Arch Intern Med 167, 2461-2468.

18. Sofi F, Cesari F, Abbate R, et al. (2008) Adherence to Mediterranean diet and health status: meta-analysis. BMJ 337 , a1344-a1350.

19. Trichopoulou A, Orfanos P, Norat T, et al. (2005) Modified Mediterranean diet and survival: EPIC-elderly prospective cohort study. BMJ 330, 991-997.

20. Trichopoulou A, Costacou T, Bamia C, et al. (2003) Adherence to a Mediterranean diet and survival in a Greek population. $N$ Engl J Med 348, 2599-2608.

21. Lagiou P, Trichopoulos D, Sandin S, et al. (2006) Mediterranean dietary pattern and mortality among young women: a cohort study in Sweden. Br J Nutr 96, 384-392.
22. Osler M \& Schroll M (1997) Diet and mortality in a cohort of elderly people in a north European community. Int $J$ Epidemiol 26, 155-159.

23. Trichopoulou A, Bamia C \& Trichopoulos D (2009) Anatomy of health effects of Mediterranean diet: Greek EPIC prospective cohort study. BMJ 338, 2337-2344.

24. Agudo A, Cabrera L, Amiano P, et al. (2007) Fruit and vegetable intakes, dietary antioxidant nutrients, and total mortality in Spanish adults: findings from the Spanish cohort of the European Prospective Investigation into Cancer and Nutrition (EPIC-Spain). Am J Clin Nutr 85, 1634-1642.

25. Riboli E \& Kaaks R (1997) The EPIC project: rationale and study design. Int J Epidemiol 26, Suppl. 1, S6-S14.

26. Riboli E, Hunt KJ, Slimani N, et al. (2002) European Prospective Investigation into Cancer and Nutrition (EPIC): study populations and data collection. Public Health Nutr 5 , $1113-1124$

27. González CA (1997) Relative validity and reproducibility of a diet history questionnaire in Spain. I. Foods. Int J Epidemiol 26, 91-99.

28. González CA (1997) Relative validity and reproducibility of a diet history questionnaire in Spain. II. Nutrients. Int J Epidemiol 26, 100-109.

29. Slimani N, Deharveng G, Unwin I, et al. (2007) The EPIC nutrient database project (ENDB): a first attempt to standardize nutrient databases across the 10 European countries participating in the EPIC study. Eur J Clin Nutr 61, $1037-1056$

30. Buckland G, Gonzalez CA, Agudo A, et al. (2009) Adherence to the Mediterranean diet and risk of coronary heart disease in the Spanish EPIC Cohort Study. Am J Epidemiol 170, $1518-1529$

31. Willett WC, Howe GR \& Kushi LH (1997) Adjustment for total energy intake in epidemiologic studies. Am J Clin Nutr $\mathbf{6 5}$, Suppl. 4, S1220-S1228.

32. Wareham NJ, Jakes RW, Rennie KL, et al. (2003) Validity and repeatability of a simple index derived from the short physical activity questionnaire used in the European Prospective Investigation into Cancer and Nutrition (EPIC) study. Public Health Nutr 6, 407-413.

33. Lunn M \& McNeil D (1995) Applying Cox regression to competing risks. Biometrics 51, 524-532.

34. Wei LJ, DY Lin \& Weissfeld L (1989) Regression analysis of multivariate incomplete failure time data by modeling marginal distributions. J Am Stat Assoc 84, 1065-1073.

35. Tong PC, Kong AP, So WY, et al. (2007) The usefulness of the International Diabetes Federation and the National Cholesterol Education Program's Adult Treatment Panel III definitions of the metabolic syndrome in predicting coronary heart disease in subjects with type 2 diabetes. Diabetes Care 30, 1206-1211.

36. Arriola L, Martinez-Camblor P, Larranaga N, et al. (2010) Alcohol intake and the risk of coronary heart disease in the Spanish EPIC cohort study. Heart 96, 124-130.

37. Goldberg GR, Black AE, Jebb SA, et al. (1991) Critical evaluation of energy intake data using fundamental principles of energy physiology: 1 . Derivation of cut-off limits to identify under-recording. Eur J Clin Nutr 45, 569-581.

38. World Cancer Research Fund \& American Institute for Cancer Research (2007) Food, Nutrition, Physical Activity and the Prevention of Cancer: A Global Perspective. Washington, DC: AICR

39. Knoops KT, de Groot LC, Kromhout D, et al. (2004) Mediterranean diet, lifestyle factors, and 10-year mortality in elderly European men and women: the HALE project. JAMA 292, $1433-1439$. 
40. Osler M, Heitmann BL, Gerdes LU, et al. (2001) Dietary patterns and mortality in Danish men and women: a prospective observational study. BrJ Nutr 85, 219-225.

41. Kouris-Blazos A, Gnardellis C, Wahlqvist ML, et al. (1999) Are the advantages of the Mediterranean diet transferable to other populations? A cohort study in Melbourne, Australia. Br J Nutr 82, 57-61.

42. Panagiotakos DB, Pitsavos C, Polychronopoulos E, et al. (2004) Can a Mediterranean diet moderate the development and clinical progression of coronary heart disease? A systematic review. Med Sci Monit 10, RA193-RA198.

43. Panagiotakos DB, Dimakopoulou K, Katsouyanni K, et al. (2009) Mediterranean diet and inflammatory response in myocardial infarction survivors. Int J Epidemiol 38, 856-866.

44. Lopez-Miranda J, Perez-Jimenez F, Ros E, et al. (2010) Olive oil and health: summary of the II international conference on olive oil and health consensus report, Jaen and Cordoba (Spain) 2008. Nutr Metab Cardiovasc Dis 20, 284-294.

45. Serra-Majem L, Roman B \& Estruch R (2006) Scientific evidence of interventions using the Mediterranean diet: a systematic review. Nutr Rev 64, Suppl. 1, S27-S47.

46. Verberne L, Bach-Faig A, Buckland G, et al. (2010) Association between the Mediterranean diet and cancer risk: a review of observational studies. Nutr Cancer 62, 860-870.
47. Barzi F, Woodward M, Marfisi RM, et al. (2003) Mediterranean diet and all-causes mortality after myocardial infarction: results from the GISSI-Prevenzione trial. Eur J Clin Nutr 57, 604-611.

48. Fernandez-Jarne E, Martinez-Losa E, Prado-Santamaria M, et al. (2002) Risk of first non-fatal myocardial infarction negatively associated with olive oil consumption: a casecontrol study in Spain. Int J Epidemiol 31, 474-480.

49. Ruiz-Canela M \& Martinez-Gonzalez MA (2011) Olive oil in the primary prevention of cardiovascular disease. Maturitas 68, 245-250.

50. Bendinelli B, Masala G, Saieva C, et al. (2011) Fruit, vegetables, and olive oil and risk of coronary heart disease in Italian women: the EPICOR Study. Am J Clin Nutr 93 275-283.

51. Gronbaek M, Becker U, Johansen D, et al. (2000) Type of alcohol consumed and mortality from all causes, coronary heart disease, and cancer. Ann Intern Med 133, 411-419.

52. Bingham SA, Gill C, Welch A, et al. (1997) Validation of dietary assessment methods in the UK arm of EPIC using weighed records, and 24-hour urinary nitrogen and potassium and serum vitamin $\mathrm{C}$ and carotenoids as biomarkers. Int J Epidemiol 26, Suppl. 1, S137-S151.

53. Livingstone MB \& Black AE (2003) Markers of the validity of reported energy intake. J Nutr 133, Suppl. 3, S895-S920. 